\title{
通过苄醇与炔烃的环化反应快速高效构建狮及螺环狮
}

\author{
余佳佳 $^{a}$ 杨 珊 ${ }^{a}$ 吴 镇 ${ }^{a}$ 朱 晨*,a,b \\ ( 苏州大学材料与化学化工学部 苏州 215123) \\ $\left({ }^{b}\right.$ 中国科学院上海有机化学研究所 天然产物有机合成重点实验室 上海 200032)
}

\begin{abstract}
摘要 作为一类重要的碳环化合物, 狮、螺环狮及其衍生物常见于各种天然产物骨架结构中, 并作为合成中间体广泛 地应用于材料、医药、有机不对称合成等领域. 在路易斯酸 $\mathrm{TiCl}_{4}$ 或 $\mathrm{AlCl}_{3}$ 作用下，二苯甲醇或芳基取代环醇等苄醇通 过生成碳正离子中间体, 与炔烃进行环化反应高效合成多种狮及螺环狮. 该反应仅需 $30 \mathrm{~min}$, 反应过程中完成了 2 个新 $\mathrm{C}-\mathrm{C}$ 键的构建, 对具有各种取代基的炔烃均有较好的适用性. 芳基取代的环丁醇、环已醇、环庚醇、环辛醇以及环十 二醇都可以适用于该方法, 多样性地构建多种有合成价值的螺环骨架. 该方法具有操作简便、反应时间短、条件温和 等优点.
\end{abstract}

关键词＼cjkstart路易斯酸; 环化反应; 茚; 螺环狮

\section{Annulation of Benzylic Alcohols with Alkynes for Rapid and Efficient Synthesis of Indenes and Spiroindenes}

\author{
Yu, Jiajia ${ }^{a} \quad$ Yang, Shan $^{a} \quad \mathrm{Wu}$, Zhen $^{a} \quad$ Zhu, Chen ${ }^{*, a, b}$
}

$\left({ }^{a}\right.$ College of Chemistry, Chemical Engineering and Materials Science, Soochow University, Suzhou 215123)

( ${ }^{b}$ Key Laboratory of Synthetic Chemistry of Natural Substances, Shanghai Institute of Organic Chemistry, Shanghai 200032)

\begin{abstract}
As a type of important carbocyclic compounds, indenes and spiroindenes are not only widely found in natural products but extensively employed as synthetic building blocks in materials, pharmaceuticals, and asymmetric synthesis. An efficient and rapid synthesis of indenes and spiroindenes via the annulation of benzylic alcohols (or aryl-substituted cycloalkanols) with alkynes in the presence of $\mathrm{TiCl}_{4}$ or $\mathrm{AlCl}_{3}$ was desclosed. This reaction is normally completed within 30 min at room temperature and compatible with a variety of substituted alkynes. Two new $\mathrm{C}-\mathrm{C}$ bonds are constructed during the reaction. Cycloalkanols such as cyclobutanol, cyclohexanol, cycloheptanol, cyclooctanol, and cyclododecanol are suitable substrates to afford a set of valuable spiroindenes. This method is featured with simple operation, short reaction time, and mild reaction conditions.
\end{abstract}

Keywords Lewis acid; cyclization; indene; spiroindene

䒢和螺环䒢是天然产物中常见的碳环骨架 ${ }^{[1,2]}$, 并 因其特有结构及性质被广泛应用于生物 ${ }^{[3]}$ 、医药 ${ }^{[4]}$ 、材 料 ${ }^{[5]}$ 等领域. 荫作为配体骨架常用于有机金属化合物的 合成中 ${ }^{[6]}$, 螺环狮由于存在螺手性常被用于合成各种手 性配体, 并在不对称合成领域发挥着重要作用 ${ }^{[7]}$. 因此, 发展一类通用的方法, 以简单易得的原料高效构建这类 结构, 具有重要的研究意义.

对于狮的合成方法有很多报道, 大致可以分为过渡 金属催化 ${ }^{[8]}$ 和路易斯酸催化 ${ }^{[9]}$ 两种方式. 其中, 路易斯
酸以其价格便宜、反应效果好而受到广泛关注. 利用路 易斯酸箱取醇羟基产生的碳正离子, 既能在分子内与芳 环进行 Friedel-Crafts 环化反应 ${ }^{\left[{ }^{d} \sim 9 \mathrm{f}\right]}$, 也可与炔烃发生分 子间加成 ${ }^{[10]}$. 而将这两种途径相结合, 利用苄位碳正离 子与炔烃进行环化, 便可构建多种狮类衍生物. 例如: 田仕凯课题组 ${ }^{[1]}$ 利用 $\mathrm{FeCl}_{3}$ 断裂茮位磺酰胺的 $\mathrm{C}-\mathrm{N}$ 键 产生芐位碳正离子，与炔烃发生分子间环化构建了狮类 化合物. 然而该反应时间较长, 且需加热到 $80{ }^{\circ} \mathrm{C}$ 进行 (图 1A). 周锡庚课题组 ${ }^{[12]}$ 利用 $\mathrm{FeCl}_{3}$ 盿取二苯甲醇的羟

\footnotetext{
* Corresponding author. E-mail: chzhu@suda.edu.cn

Received August 10, 2018; revised September 10, 2018; published online October 12, 2018.

Dedicated to Professor Qingyun Chen on the occasion of his 90th birthday.

Project supported by the National Natural Science Foundation of China (No. 21722205).

国家自然科学基金(No. 21722205)资助项目.
} 
基形成苄位碳正离子，与炔烃环化得到狮类化合物. 但 是反应在此条件下容易过度进行, 产生的碳正离子会再 次与产物狮发生 Friedel-Crafts 反应(图 1B).

\section{Previous work}

(A)<smiles>[R]C#C[R]</smiles>

(B)

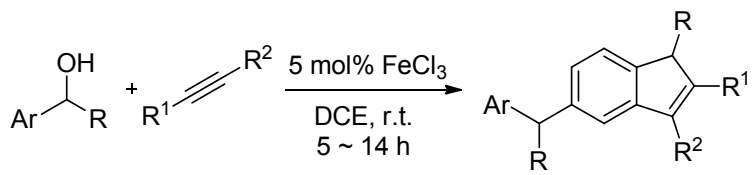

(C) This work<smiles>OC(c1ccccc1)c1ccccc1</smiles><smiles>[R]C#C[O]</smiles><smiles></smiles>
r.t., $30 \mathrm{~min}$<smiles>[R]C1=C([R])C(c2ccccc2)c2ccccc21</smiles><smiles>[R]C#C[R]</smiles>

- Short reaction time $\mathrm{O}$ Mild conditions

Two $\mathrm{C}-\mathrm{C}$ bonds formation

图 1 路易斯酸促进的碳正离子与炔烃的环化反应

Figure 1 Lewis acid-promoted cyclization of carbocation with alkynes

由上可见，目前报道的路易斯酸催化合成狮环的方 法仍有一些不足，且利用其合成螺环狮更是鲜有报 道 ${ }^{[13]}$. 我们课题组 ${ }^{[14,15]}$ 以芳基取代的环醇为原料, 开展 了系统性的有机开环反应的研究. 基于前期工作的启 发，在本工作中我们利用芳基取代的环醇在路易斯酸 $\mathrm{TiCl}_{4}$ 或 $\mathrm{AlCl}_{3}$ 的促进下生产的碳正离子中间体，与炔烃 发生环化反应，从而快速高效地合成了一系列螺环化合 物. 该方法反应时间短、条件温和、操作简单, 反应中 完成了 2 个新 $\mathrm{C}-\mathrm{C}$ 键的构建, 对具有各种取代基的炔 烃均有较好的适用性. 通过改变环醇的碳环大小或者结 构, 反应能灵活地在产物中引入不同的碳环, 构建了多 种含中环以及大环复杂骨架结构的螺环化合物(图 1C).

\section{1 结果与讨论}

\section{1 反应条件优化}

室温下, 以二苯甲醇 $(\mathbf{1 a})$ 和二苯乙炔 $(\mathbf{2 b})$ 作为底物, $20 \mathrm{~mol} \% \mathrm{AlCl}_{3}$ 为路易斯酸, 干燥的二氯甲烷 (DCM) 为 溶剂, 进行反应条件探究. 考虑到溶剂中的残留水分容 易使路易斯酸发生分解，首先篎选了不同的干燥剂(表 1, Entries 1 3). 相比于常规干燥剂硫酸钠和硫酸镁, 反应中加入 $100 \mathrm{mg} 4 \AA$ 分子耖可使产率达到 $52 \%$. 接着,
当将 $4 \AA$ 分子篮的用量逐步增加到 $400 \mathrm{mg}$ 时, 产率达到 了 $68 \%$ (表 1, Entries 4,5). 而当考察其他路易斯酸的反 应情况时(表 1, Entries 6 12), 惊喜地发现在 $\mathrm{TiCl}_{4}$ 条件 下，反应仅需 $30 \mathrm{~min}$ ，产率达到了 $87 \%$. 接下来，考察 溶剂对反应的影响(表 1, Entries 13 18), 结果显示 $\mathrm{CHCl}_{3}$ 和 $\mathrm{C}_{6} \mathrm{H}_{5} \mathrm{~F}$ 也能以较优的产率得到目标产物. 最后, 分别以 1.1 equiv. 的 $\mathrm{TiCl}_{4}$ 和 $\mathrm{AlCl}_{3}$ 尝试反应, 均得到很高 的产率(表 1, Entries 19，20). 考虑到反应的绿色和经济 性, 最终我们确定表 1 中的 Entry 12 为最优条件.

表 1 狮的反应条件优化 ${ }^{a}$

Table 1 Optimization of reaction conditions for indene ${ }^{a}$

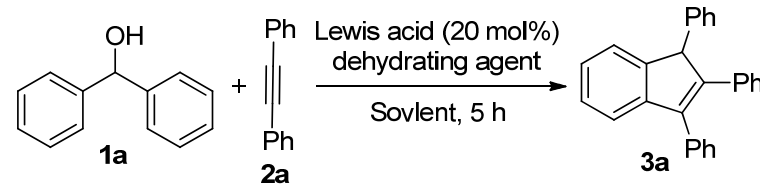

\begin{tabular}{cllcc}
\hline Entry & Lewis acid & Dehydrating agent & Solvent & Yield $^{b} \%$ \\
\hline 1 & $\mathrm{AlCl}_{3}$ & $\mathrm{Na}_{2} \mathrm{SO}_{4}(2.0$ equiv. $)$ & $\mathrm{DCM}$ & 23 \\
2 & $\mathrm{AlCl}_{3}$ & $\mathrm{MgSO}_{4}(2.0$ equiv. $)$ & $\mathrm{DCM}$ & 29 \\
$3^{c}$ & $\mathrm{AlCl}_{3}$ & $4 \AA \mathrm{MS}$ & $\mathrm{DCM}$ & 52 \\
$4^{d}$ & $\mathrm{AlCl}_{3}$ & $4 \AA \mathrm{MS}$ & $\mathrm{DCM}$ & 63 \\
5 & $\mathrm{AlCl}_{3}$ & $4 \AA \mathrm{MS}$ & $\mathrm{DCM}$ & 68 \\
6 & $\mathrm{AgOTf}$ & $4 \AA \mathrm{MS}$ & $\mathrm{DCM}$ & $<5$ \\
7 & $\mathrm{Sc} \mathrm{OTf})_{3}$ & $4 \AA \mathrm{MS}$ & $\mathrm{DCM}$ & 55 \\
8 & $\left.\mathrm{Gd}_{(\mathrm{OTf}}\right)_{3}$ & $4 \AA \mathrm{MS}$ & $\mathrm{DCM}$ & $<5$ \\
9 & $\mathrm{BF}_{3} \cdot \mathrm{Et}_{2} \mathrm{O}$ & $4 \AA \mathrm{MS}$ & $\mathrm{DCM}$ & 65 \\
10 & $\mathrm{FeCl}_{3}$ & $4 \AA \mathrm{MS}$ & $\mathrm{DCM}$ & 85 \\
11 & $\mathrm{ZnCl}_{2}$ & $4 \AA \mathrm{MS}$ & $\mathrm{DCM}$ & 0 \\
$12^{e}$ & $\mathrm{TiCl}_{4}$ & $4 \AA \mathrm{MS}$ & $\mathrm{DCM}$ & 87 \\
$13^{e}$ & $\mathrm{TiCl}_{4}$ & $4 \AA \mathrm{MS}$ & $\mathrm{CH} \mathrm{CN}_{3}$ & 0 \\
$14^{e}$ & $\mathrm{TiCl}_{4}$ & $4 \AA \mathrm{MS}$ & $\mathrm{CHCl}$ & 84 \\
$15^{e}$ & $\mathrm{TiCl}_{4}$ & $4 \AA \mathrm{MS}$ & $\mathrm{C}_{6} \mathrm{H}_{5} \mathrm{~F}$ & 83 \\
$16^{e}$ & $\mathrm{TiCl}_{4}$ & $4 \AA \mathrm{MS}$ & $\mathrm{DMF}$ & 0 \\
$17^{e}$ & $\mathrm{TiCl}_{4}$ & $4 \AA \mathrm{MS}$ & $\mathrm{THF}$ & 0 \\
$18^{e}$ & $\mathrm{TiCl}_{4}$ & $4 \AA \mathrm{MS}$ & $\mathrm{DCE}$ & 0 \\
$19^{e, f}$ & $\mathrm{TiCl}_{4}$ & $4 \AA \mathrm{MS}$ & $\mathrm{DCM}$ & 88 \\
$20^{e, g}$ & $\mathrm{AlCl}_{3}$ & $4 \AA \mathrm{MS}$ & $\mathrm{DCM}$ & 90 \\
\hline
\end{tabular}

${ }^{a}$ Reaction conditions: 1a $(0.2 \mathrm{mmol}), \mathbf{2 a}(0.2 \mathrm{mmol}), 4 \AA \mathrm{MS}(400 \mathrm{mg})$, and Lewis acid $(20 \mathrm{~mol} \%)$ in $2 \mathrm{~mL}$ of DCM at r.t., $5 \mathrm{~h} .{ }^{b}$ Yields of isolated products. ${ }^{c} 100 \mathrm{mg}$ of $4 \AA$ MS was used. ${ }^{d} 300 \mathrm{mg}$ of $4 \AA$ MS was used. ${ }^{e}$ Reaction time: $30 \mathrm{~min} .{ }^{f} 0.22 \mathrm{mmol}$ of $\mathrm{TiCl}_{4} .{ }^{g} 0.22 \mathrm{mmol}$ of $\mathrm{AlCl}_{3}$.

\section{2 狮的合成}

在得到最优反应条件后，对反应适用性进行考察. 首先，改变炔烃两侧苯环的取代基. 从表 2 中可以看出, 取代基为给电子基例如对甲氧基和对叔丁基时，反应均 有较好的兼容性(表 $2,3 \mathbf{b}, 3 \mathbf{c}$ ). 当取代基为卤素时，氟代 和氯代产物在增加 $\mathrm{TiCl}_{4}$ 用量的情况下可以得到不错的 产率(表 2，3d，3e)，溴代产物也能获得中等收率(表 2 , 3f). 另外, 苯环间位被氟取代时, 反应也能顺利进行(表 2, 3g). 该方法还可兼容脂肪炔烃，并以良好的收率得 到产物(表 2, 3h). 最后，我们尝试了单取代炔烃的反应， 
但未能得到对应的产物.

表 2 二苯甲醇与炔烃合成狮 ${ }^{a, b}$

Table 2 Synthesis of indenes from benzhydrol and alkynes<smiles>[R]C#C[R]</smiles><smiles>[R]c1ccc(C2=C(c3ccc([R])cc3)C(c3ccccc3)c3ccccc32)cc1</smiles>

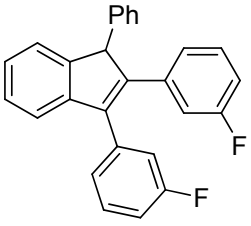

3g: $34 \%^{c}$

3a: $\mathrm{R}=\mathrm{H}, 87 \%$

3b: $R=O M e, 64 \%$

3c: $\mathrm{R}={ }^{t} \mathrm{Bu}, \quad 98 \%$

3d: $R=F, \quad 53 \%^{c}$

3e: $\mathrm{R}=\mathrm{Cl}, \quad 61 \%^{\mathrm{c}}$

3f: $\mathrm{R}=\mathrm{Br}, \quad 45 \%$

Reaction conditions: 1a $(0.2 \mathrm{mmol}), 2(0.2 \mathrm{mmol}, 1.0$ equiv. $), \mathrm{TiCl}_{4}(0.04$ $\mathrm{mmol}, 20 \mathrm{~mol} \%)$, and $4 \AA \mathrm{MS}(400 \mathrm{mg})$ in $\operatorname{dry}$ DCM $(2.0 \mathrm{~mL})$ at r.t.. ${ }^{b}$ Yield of isolated products. ${ }^{c} 1.1$ equiv. of $\mathrm{TiCl}_{4}$.

\section{3 螺环狮的合成}

接着我们将研究重点转向螺环狮的合成. 以 1-苯基 环丁醇作为底物, 在 1.1 equiv. $\mathrm{TiCl}_{4}$ 的作用下, 尝试与 炔烃发生环化反应. 值得一提的是, 环丁醇是具有较大 环张力的碳环结构 ${ }^{[16]}$, 而在该反应条件下碳环未发生 开环, 被成功地引入到底物中. 反应仅用 $30 \mathrm{~min}$, 就以 $89 \%$ 的产率得到了对应的螺环茚(表 3, 5a). 之后，对反 应底物的普适性进行了研究. 考虑到芳基澳化物可以进 行交叉偶联反应而显现出重要的应用价值, 我们首先考 察了对溴苯基环丁醇的反应情况. 反应能以较好的收率 得到相应的产物(表 3, 5b) , 而芳基溴的保留可以为后期 的产物修饰提供平台. 在炔烃的苯环对位安装上甲氧基 时，反应可以中等收率得到产物(表 $3, \mathbf{5 c}$ ). 然而，当苯 环的对位和间位分别连接卤素时, 需用 1.1 equiv.的 $\mathrm{AlCl}_{3}$ 替换 $\mathrm{TiCl}_{4}$ 才能得到较好的产率(表 $3, \mathbf{5 d} \sim \mathbf{5 g}$ ). 该 反应底物并不局限于芳基炔烃, 脂肪链取代的 3-己炔也 同样适用于该反应，以较高产率得到相应的产物(表 3 , 5h). 但该方法并不适用于端基炔的底物. 延长反应时 间至 $1 \mathrm{~h}$, 该方法也能适用于杂芳基环丁醇(表 3, 5i). 我 们继续考察了 1-芳基环己醇的反应情况. 从表 3 中看到, 改变底物中脂肪环或者芳基的结构能得到多种对应的

表 3 芳基取代环醇与炔烃合成螺环狮 $a, b$

Table 3 Synthesis of spiroindenes from 1-aryl cycloalkanols and alkynes

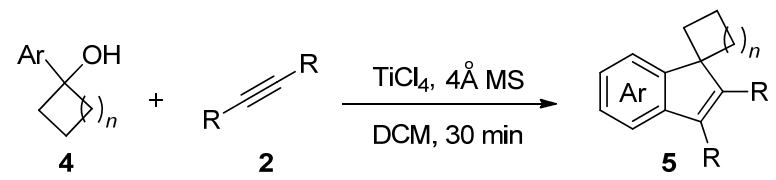

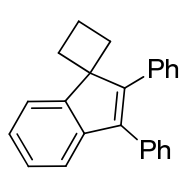

5a: $89 \%$<smiles>Fc1cccc(C2=C(c3cccc(F)c3)C3(CCC3)c3ccccc32)c1</smiles>

5g: $42 \%^{c}$<smiles>CC1(C)CC(C)(C)CC2(C1)C(c1ccccc1)=C(c1ccccc1)c1ccccc12</smiles>

5m: $46 \%$<smiles>Brc1ccc2c(c1)C(c1ccccc1)C(c1ccccc1)C21CCC1</smiles>

5b: $67 \%$<smiles>CCC1=C(CC)C2(CCC2)c2ccccc21</smiles>

5h: $64 \%$<smiles></smiles>

5n: $93 \%$<smiles>COc1ccc(C2=C(c3ccc(OC)cc3)C3(CCCC3)c3ccccc32)cc1</smiles>

5c: $43 \%$<smiles></smiles>

5i: $35 \%^{d}$<smiles>c1ccc(C2=C(c3ccccc3)C3(CCCCC3)c3ccc4ccccc4c32)cc1</smiles>

5o: $60 \%$

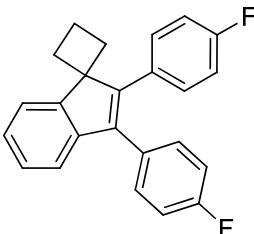

5d: $52 \%^{c}$<smiles>c1ccc(C2=C(c3ccccc3)C3(CCCCC3)c3ccccc32)cc1</smiles>

5j: $80 \%$<smiles>c1ccc(C2=C(c3ccccc3)C3(c4ccccc4)CCCCC[C-]23)cc1</smiles>

5p: $77 \%$

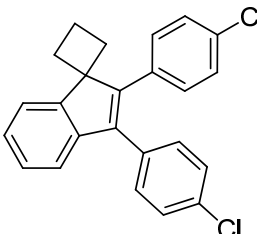

5e: $51 \%^{c}$<smiles>c1ccc(C2=C(c3ccccc3)C3(CCC(c4ccccc4)CC3)c3ccccc32)cc1</smiles>

5k: $82 \%$<smiles>[C+](C1=C(c2ccccc2)C2(CCCCCCC2)c2ccccc21)c1ccccc1</smiles>

$5 q: 62 \%$

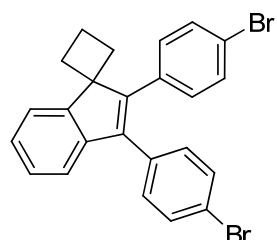

5f: $73 \%^{c}$<smiles>CC1(C)CCC2(CC1)C(c1ccccc1)=C(c1ccccc1)c1ccccc12</smiles>

5I: $87 \%$

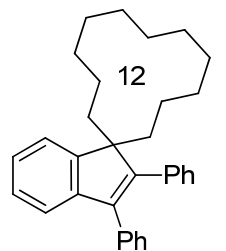

5r: $91 \%$

${ }^{a}$ Conditions: 4 (0.2 mmol), 2 (0.2 mmol, 1.0 equiv.), TiCl $_{4}\left(0.22 \mathrm{mmol}, 1.1\right.$ equiv.), and $4 \AA \mathrm{MS}(400 \mathrm{mg})$ in dry DCM (2.0 mL) at r.t. ${ }^{b}$ Yield of isolated products.

${ }^{c} 1.1$ equiv. of $\mathrm{AlCl}_{3}$ instead of $\mathrm{TiCl}_{4} \cdot{ }^{d}$ Reaction time is $1 \mathrm{~h}$. 
螺环产物，甚至对于大位置的金刚烷也有很好的兼容性 (表 $3, \mathbf{5 j} \sim 5 \mathbf{5 0}$ ). 更令人欣喜的是, 环庚醇、环辛醇以及环 十二醇通过该反应均能以很好的产率构建含中环及大 环结构的螺环䒢(表 3, 5p 5r). 但是 1-芳基环戊醇在该 反应中不能兼容, 主要生成了分子内脱水的副产物.

根据已有的实验结果, 我们提出可能的反应机理 (图 2). 首先, 1-芳基环丁醇 $\mathbf{4}$ 的羟基被路易斯酸 $\mathrm{TiCl}_{4}$ 箱 取, 形成苄位碳正离子 $\mathbf{I}$. 中间体 $\mathbf{I}$ 与炔烃加成产生烯基 正离子 II. 中间体 II 与芳环发生分子内亲电环化反应得 到芳基正离子 III. 最后, 中间体 III 脱氢芳构化得到螺 环产物 5.

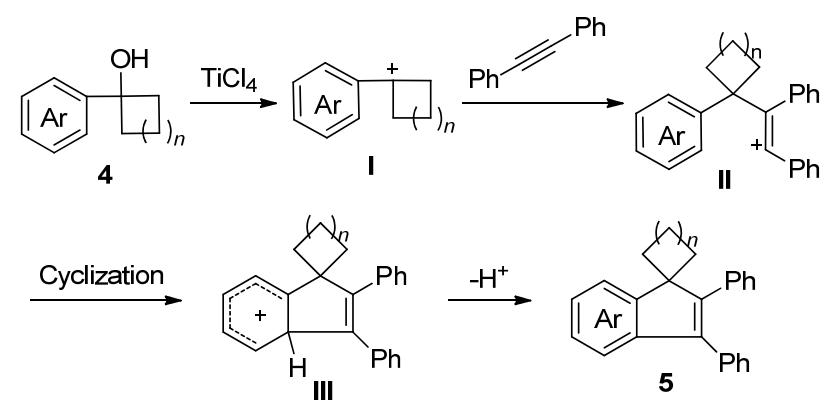

图 2 反应机理

Figure 2 Proposed reaction mechanism

\section{2 结论}

在路易斯酸 $\mathrm{TiCl}_{4}$ 或 $\mathrm{AlCl}_{3}$ 作用下，二苯甲醇或芳基 取代环醇等苄醇通过生成碳正离子中间体，与炔烃发生 环化反应高效合成多种狮及螺环狮. 实验结果表明, 具 有较大张力的环丁醇未在该反应条件下发生开环断裂, 其碳环结构被成功地引入到产物中. 通过这种策略, 仅 需改变底物的碳环结构, 就可以灵活地构建多种含中环 以及大环骨架结构的螺环产物. 反应在室温下仅需 30 $\min$ 即可完成. 反应过程中完成了 2 个新 $\mathrm{C}-\mathrm{C}$ 键的构 建, 对具有各种取代基的炔烃均有较好的适用性.

\section{3 实验部分}

\section{1 仪器与试剂}

Bruker 公司 $400 \mathrm{MHz}$ AVANCE III HD 核磁共振仪, 溶剂 $\mathrm{CDCl}_{3}$, 内标 TMS; BRUKER 公司 VERTEX 70 傅 里叶变换红外光谱仪; Waters Micromass 公司 GCT Premier 高分辨质谱仪; INESA WRR 显微熔点仪测定.

实验用的所有试剂均为市售试剂, 没有经过进一步 纯化. 二氯甲烷 $(\mathrm{DCM})$ 以氢化钙 $\left(\mathrm{CaH}_{2}\right)$ 除水, 经蒸馏收 集使用. $4 \AA \mathrm{MS}$ 分子篮经研磨及 $300{ }^{\circ} \mathrm{C}$ 高温烘焙处理. 所有已知产物都经核磁氢谱、碳谱和液质联用表征并与 文献数据对比确认结构. 所有未知产物都经核磁氢谱、
碳谱、红外和高分辨质谱表征确定结构.

\section{2 实验方法}

\subsection{1 狮类化合物 $\mathbf{3}$ 的合成}

在 $5 \mathrm{~mL}$ 的反应瓶中, 依次加入 $0.2 \mathrm{mmol}$ 二苯甲醇 (1a), $0.2 \mathrm{mmol}$ 二芳基乙炔(2), $400 \mathrm{mg} 4 \AA \mathrm{MS}$ 和 $2.0 \mathrm{~mL}$ $\mathrm{DCM}$, 然后加入 $0.04 \mathrm{mmol}$ (或 $0.22 \mathrm{mmol}$ ) $\mathrm{TiCl}_{4}$. 室温下 搅拌 $30 \mathrm{~min}$. 反应结束后, 加水猝灭, DCM 萃取, 有机 层用无水硫酸钠干燥, 减压旋干溶剂, 经柱层析分离得 到目标产物 3.

1,2,3-三苯基- $1 H$-狮(3a): 白色固体，产率 87\%. m.p. $132 \sim 134{ }^{\circ} \mathrm{C}$ (文献值 ${ }^{[11]}$ m.p. $133 \sim 135{ }^{\circ} \mathrm{C}$ ); ${ }^{1} \mathrm{H}$ NMR $\left(400 \mathrm{MHz}, \mathrm{CDCl}_{3}\right) \delta: 7.44 \sim 7.37(\mathrm{~m}, 4 \mathrm{H}), 7.36 \sim 7.32(\mathrm{~m}$, $1 \mathrm{H}), 7.30 \sim 7.21(\mathrm{~m}, 3 \mathrm{H}), 7.21 \sim 7.16(\mathrm{~m}, 2 \mathrm{H}), 7.16 \sim 7.13$ (m, 3H), $7.13 \sim 7.08(\mathrm{~m}, 3 \mathrm{H}), 7.07 \sim 6.98(\mathrm{~m}, 3 \mathrm{H}), 5.10(\mathrm{~s}$, $1 \mathrm{H}) ;{ }^{13} \mathrm{C}$ NMR $\left(100 \mathrm{MHz}, \mathrm{CDCl}_{3}\right) \delta: 147.8,145.2,144.6$, $140.4,139.4,135.2,135.1,129.1,128.9,128.3,128.3$, $127.8,127.4,127.1,126.5,126.3,126.2,125.3,123.5$, 120.1, 57.6; FT-IR (film) v: 3079, 3019, 2925, 2857, 1492, $1027 \mathrm{~cm}^{-1}$; HRMS [APCI] calcd for $\mathrm{C}_{27} \mathrm{H}_{21}[\mathrm{M}+\mathrm{H}]^{+}$ 345.1638 , found 345.1633 .

2,3-二(4-甲氧基苯基)-1-苯基- $1 H$-茚(3b)：白色固 体, 产率 64\%. m.p. 127 129 ${ }^{\circ} \mathrm{C}$; ${ }^{1} \mathrm{H}$ NMR $(400 \mathrm{MHz}$, $\left.\mathrm{CDCl}_{3}\right) \delta: 7.43 \sim 7.39(\mathrm{~m}, 2 \mathrm{H}), 7.31 \sim 7.27(\mathrm{~m}, 3 \mathrm{H})$, $7.26 \sim 7.22(\mathrm{~m}, 2 \mathrm{H}), 7.21 \sim 7.15(\mathrm{~m}, 4 \mathrm{H}), 7.14 \sim 7.09(\mathrm{~m}$, $2 \mathrm{H}), 7.04 \sim 7.00(\mathrm{~m}, 2 \mathrm{H}), 6.69 \sim 6.64(\mathrm{~m}, 2 \mathrm{H}), 5.09(\mathrm{~s}$, $1 \mathrm{H}), 3.91$ (s, 3H), 3.73 (s, 3H); ${ }^{13} \mathrm{C}$ NMR $(100 \mathrm{MHz}$, $\left.\mathrm{CDCl}_{3}\right) \delta: 158.4,157.7,147.6,144.9,144.2,139.8,138.6$, $130.3,123.0,128.2,128.1,127.7,127.6,126.4,126.1$, 124.9, 123.3, 119.7, 113.8, 112.9, 57.4, 54.8, 54.5; FT-IR (film) $v: 3060,2956,1513,1244,1176,1029 \mathrm{~cm}^{-1}$; HRMS [APCI] calcd for $\mathrm{C}_{29} \mathrm{H}_{25} \mathrm{O}_{2}[\mathrm{M}+\mathrm{H}]^{+} 405.1849$, found 405.1859 .

2,3-二(4-(叔丁基)苯基)-1-苯基- $1 H$-狮(3c)：白色固 体, 产率 98\%. m.p. $114 \sim 115{ }^{\circ} \mathrm{C} ;{ }^{1} \mathrm{H}$ NMR $(400 \mathrm{MHz}$, $\left.\mathrm{CDCl}_{3}\right) \delta: 7.47(\mathrm{~d}, J=8.0 \mathrm{~Hz}, 2 \mathrm{H}), 7.39(\mathrm{~d}, J=8.0 \mathrm{~Hz}$, 2H), $7.25 \sim 7.19(\mathrm{~m}, 7 \mathrm{H}), 7.18 \sim 7.13(\mathrm{~m}, 2 \mathrm{H}), 7.12 \sim 7.05$ (m, 4H), 4.98 (s, 1H), 1.30 (s, 9H), 1.11 (s, 9H); ${ }^{13} \mathrm{C}$ NMR $\left(100 \mathrm{MHz}, \mathrm{CDCl}_{3}\right) \delta: 149.8,148.9,147.7,145.1,143.9$, $140.1,140.0,132.4,132.0,128.6,128.2,128.2,127.6$, $126.3,126.0,125.1,125.0,124.3,123.2,120.1,57.4,34.2$, 33.9, 31.0, 30.7; FT-IR (film) v: 2961, 2864, 1684, 1508, $1363,1268 \mathrm{~cm}^{-1}$; HRMS [APCI] calcd for $\mathrm{C}_{35} \mathrm{H}_{37}[\mathrm{M}+\mathrm{H}$ ] ${ }^{+} 457.2890$, found 457.2889 .

2,3-二(4-氟苯基)-1-苯基- $1 H$-茚(3d)：白色固体，产 率 53\%. m.p. $144 \sim 145{ }^{\circ} \mathrm{C} ;{ }^{1} \mathrm{H}$ NMR $\left(400 \mathrm{MHz}, \mathrm{CDCl}_{3}\right)$ 
$\delta: 7.43 \sim 7.35(\mathrm{~m}, 2 \mathrm{H}), 7.30 \sim 7.25(\mathrm{~m}, 3 \mathrm{H}), 7.24 \sim 7.18$ (m, 3H), $7.18 \sim 7.09(\mathrm{~m}, 5 \mathrm{H}), 7.09 \sim 7.02(\mathrm{~m}, 2 \mathrm{H}), 6.84 \sim$ $6.75(\mathrm{~m}, 2 \mathrm{H}), 5.06(\mathrm{~s}, 1 \mathrm{H}) ;{ }^{13} \mathrm{C} \mathrm{NMR}\left(100 \mathrm{MHz}, \mathrm{CDCl}_{3}\right) \delta$ : $162.7(\mathrm{~d}, J=62.5 \mathrm{~Hz}), 160.2(\mathrm{~d}, J=62.9 \mathrm{~Hz}), 147.5,144.5$, 144.2, 139.2, 138.9, 130.9 (d, $J=3.4 \mathrm{~Hz}), 130.8$ (d, $J=3.1$ $\mathrm{Hz}), 130.7$ (d, $J=7.9 \mathrm{~Hz}), 130.4$ (d, $J=7.9 \mathrm{~Hz}), 128.3$, 127.7, 126.6, 126.4, 125.5, 123.6, 119.8, 115.4 (d, $J=21.4$ $\mathrm{Hz}), 114.6(\mathrm{~d}, J=21.4 \mathrm{~Hz}), 57.7 ;{ }^{19} \mathrm{~F}$ NMR $(376 \mathrm{MHz}$, $\left.\mathrm{CDCl}_{3}\right) \delta:-114.0(\mathrm{~s}),-114.7$ (s); FT-IR (film) v: 3077, 3020, 1598, 1499, 1349, $1221 \mathrm{~cm}^{-1}$; HRMS [APCI] calcd for $\mathrm{C}_{27} \mathrm{H}_{19} \mathrm{~F}_{2}[\mathrm{M}+\mathrm{H}]^{+}$381.1455, found 381.1460.

2,3-二(4-氯苯基)-1-苯基- $1 H$-狮(3e)：白色固体，产 率 61\%. m.p. $145 \sim 148{ }^{\circ} \mathrm{C} ;{ }^{1} \mathrm{H}$ NMR (400 MHz, $\left.\mathrm{CDCl}_{3}\right)$ $\delta: 7.46 \sim 7.40(\mathrm{~m}, 2 \mathrm{H}), 7.38 \sim 7.29(\mathrm{~m}, 3 \mathrm{H}), 7.28 \sim 7.27$ (m, 1H), $7.26 \sim 7.24(\mathrm{~m}, 1 \mathrm{H}), 7.24 \sim 7.14(\mathrm{~m}, 4 \mathrm{H}), 7.14 \sim$ 7.06 (m, 3H), $7.06 \sim 6.99$ (m, 3H), $5.05(\mathrm{~s}, 1 \mathrm{H}) ;{ }^{13} \mathrm{C} \mathrm{NMR}$ $\left(100 \mathrm{MHz}, \mathrm{CDCl}_{3}\right) \delta: 148.0,144.9,144.2,140.1,139.1$, 133.7, 133.6, 133.6, 132.8, 130.8, 130.4, 129.2, 128.8, $128.3,128.1,127.1,126.9,126.1,124.1,120.4,58.0$; FT-IR (film) v: 3080, 2988, 1456, 1397, 1089, $1012 \mathrm{~cm}^{-1}$; HRMS [APCI] calcd for $\mathrm{C}_{27} \mathrm{H}_{19} \mathrm{Cl}_{2}[\mathrm{M}+\mathrm{H}]^{+} 413.0864$, found 413.0876.

2,3-二(4-溴苯基)-1-苯基- $1 H$-茚(3f)：白色固体, 产 率 45\%. m.p. $158 \sim 159{ }^{\circ} \mathrm{C} ;{ }^{1} \mathrm{H}$ NMR (400 MHz, $\mathrm{CDCl}_{3}$ ) $\delta: 7.66 \sim 7.53(\mathrm{~m}, 2 \mathrm{H}), 7.35 \sim 7.28(\mathrm{~m}, 5 \mathrm{H}), 7.27 \sim 7.21$ (m, $5 \mathrm{H}), 7.20 \sim 7.15(\mathrm{~m}, 1 \mathrm{H}), 7.15 \sim 7.09(\mathrm{~m}, 2 \mathrm{H}), 7.04 \sim$ $6.92(\mathrm{~m}, 2 \mathrm{H}), 5.08(\mathrm{~s}, 1 \mathrm{H}) ;{ }^{13} \mathrm{C} \mathrm{NMR}\left(100 \mathrm{MHz}, \mathrm{CDCl}_{3}\right) \delta$ : $148.1,145.0,144.2,140.2,139.1,134.1,134.1,132.1$, $131.3,131.1,130.8,128.8,128.1,127.1,126.9,126.2$, 124.1, 121.8, 121.1, 120.4, 58.0; FT-IR (film) v: 3061, 3024, 1585 1493, 1183, $1029 \mathrm{~cm}^{-1}$; HRMS [APCI] calcd for $\mathrm{C}_{27} \mathrm{H}_{19} \mathrm{Br}_{2}[\mathrm{M}+\mathrm{H}]^{+}$500.9853, found 500.9865.

2,3-二(3-氟苯基)-1-苯基- $1 H$-茚 $(\mathbf{3 g}$ )：白色固体，产 率 34\%. m.p. $110 \sim 111{ }^{\circ} \mathrm{C} ;{ }^{1} \mathrm{H}$ NMR $\left(400 \mathrm{MHz}, \mathrm{CDCl}_{3}\right.$ ) $\delta: 7.45 \sim 7.37(\mathrm{~m}, 1 \mathrm{H}), 7.30 \sim 7.27(\mathrm{~m}, 2 \mathrm{H}), 7.27 \sim 7.26$ (m, $1 \mathrm{H}), 7.24 \sim 7.11(\mathrm{~m}, 8 \mathrm{H}), 7.10 \sim 7.02(\mathrm{~m}, 2 \mathrm{H}), 6.89 \sim$ $6.85(\mathrm{~m}, 1 \mathrm{H}), 6.81 \sim 6.73(\mathrm{~m}, 2 \mathrm{H}), 5.07(\mathrm{~s}, 1 \mathrm{H}) ;{ }^{13} \mathrm{C} \mathrm{NMR}$ $\left(150 \mathrm{MHz}, \mathrm{CDCl}_{3}\right) \delta: 163.5(\mathrm{~d}, J=113.1 \mathrm{~Hz}), 161.9(\mathrm{~d}$, $J=111.5 \mathrm{~Hz}), 148.0,145.0$ (d, $J=2.1 \mathrm{~Hz}), 144.1,140.5$ (d. $J=1.2 \mathrm{~Hz}), 139.0,137.4(\mathrm{~d}, J=8.3 \mathrm{~Hz}), 137.3(\mathrm{~d}, J=8.3$ $\mathrm{Hz}), 130.4$ (d, $J=8.4 \mathrm{~Hz}), 129.4$ (d, $J=8.4 \mathrm{~Hz}), 128.8$, $128.1,127.1,126.9,126.2,125.2(\mathrm{~d}, J=2.7 \mathrm{~Hz}), 125.0$ (d, $J=2.6 \mathrm{~Hz}), 124.0,120.5,116.3(\mathrm{~d}, J=21.5 \mathrm{~Hz}), 115.9$ (d, $J=21.9 \mathrm{~Hz}), 114.8(\mathrm{~d}, J=21.0 \mathrm{~Hz}), 113.9(\mathrm{~d}, J=21.0 \mathrm{~Hz})$, $58.1 ;{ }^{19} \mathrm{~F}$ NMR $\left(376 \mathrm{MHz}, \mathrm{CDCl}_{3}\right) \delta:-112.5(\mathrm{~s})$,
-113.4 (s); FT-IR (film) v: 3065, 3026, 1947 1597, 1480, $1263 \mathrm{~cm}^{-1}$. HRMS [APCI] calcd for $\mathrm{C}_{27} \mathrm{H}_{19} \mathrm{~F}_{2}[\mathrm{M}+\mathrm{H}]^{+}$ 381.1455 , found 381.1460 .

2,3-二乙基-1-苯基- $1 H$ - 狮(3h)：无色液体. 产率 $63 \% .{ }^{1} \mathrm{H}$ NMR $\left(400 \mathrm{MHz}, \mathrm{CDCl}_{3}\right) \delta: 7.33 \sim 7.26(\mathrm{~m}, 2 \mathrm{H})$, $7.25 \sim 7.21(\mathrm{~m}, 2 \mathrm{H}), 7.21 \sim 7.17(\mathrm{~m}, 1 \mathrm{H}), 7.14 \sim 7.10(\mathrm{~m}$, 1H), 7.05 (ddd, $J=7.2,7.2,1.2 \mathrm{~Hz}, 1 \mathrm{H}), 7.03 \sim 6.97(\mathrm{~m}$, 2H), $4.43(\mathrm{~s}, 1 \mathrm{H}), 2.59$ (q, $J=7.6 \mathrm{~Hz}, 2 \mathrm{H}), 2.50 \sim 2.40(\mathrm{~m}$, $1 \mathrm{H}), 2.06 \sim 1.96(\mathrm{~m}, 1 \mathrm{H}), 1.22(\mathrm{t}, J=7.6 \mathrm{~Hz}, 3 \mathrm{H}), 1.01(\mathrm{t}$, $J=7.6 \mathrm{~Hz}, 3 \mathrm{H}) ;{ }^{13} \mathrm{C}$ NMR $\left(100 \mathrm{MHz}, \mathrm{CDCl}_{3}\right) \delta: 147.9$, $147.4,145.0,134.0,138.3,128.1,127.8,126.1,126.1$, 123.8, 123.2, 118.0, 56.1, 19.3, 18.0, 14.3, 13.5; FT-IR (film) $v$ : 3061, 3029, 1716,1599, 1448, $1064 \mathrm{~cm}^{-1}$; HRMS [APCI] calcd for $\mathrm{C}_{19} \mathrm{H}_{21}[\mathrm{M}+\mathrm{H}]^{+}$249.1638, found 249.1639 .

\section{2 .2 螺环化合物 $\mathbf{5}$ 的合成}

在 $5 \mathrm{~mL}$ 的反应瓶中, 依次加入 $0.2 \mathrm{mmol}$ 芳基环醇 (4a), $0.2 \mathrm{mmol}$ 二芳基乙炔(2), $400 \mathrm{mg} 4 \AA \mathrm{MS}$ 和 $2.0 \mathrm{~mL}$ $\mathrm{DCM}$, 然后加入 $0.22 \mathrm{mmol} \mathrm{TiCl}_{4}$ 或 $\mathrm{AlCl}_{3}$. 室温下搅拌 $30 \mathrm{~min}$. 反应结束后, 加水猝灭, 加 DCM 萃取, 有机层 用无水硫酸钠干燥, 减压旋干溶剂, 经柱层析分离得到 目标产物 5.

2',3'-二苯基螺 [环丁烷-1,1'-狮](5a): 白色固体，产 率 89\%. m.p. $131 \sim 133{ }^{\circ} \mathrm{C} ;{ }^{1} \mathrm{H}$ NMR (400 MHz, $\mathrm{CDCl}_{3}$ ) $\delta: 7.88 \sim 7.82(\mathrm{~m}, 1 \mathrm{H}), 7.41 \sim 7.35(\mathrm{~m}, 4 \mathrm{H}), 7.35 \sim 7.30$ (m, $6 \mathrm{H}), 7.29 \sim 7.22(\mathrm{~m}, 3 \mathrm{H}), 2.74 \sim 2.63(\mathrm{~m}, 2 \mathrm{H}), 2.63 \sim$ $2.52(\mathrm{~m}, 2 \mathrm{H}), 2.31 \sim 2.15(\mathrm{~m}, 1 \mathrm{H}), 1.90 \sim 1.77(\mathrm{~m}, 1 \mathrm{H})$; ${ }^{13} \mathrm{C}$ NMR (100 MHz, $\left.\mathrm{CDCl}_{3}\right) \delta: 152.3,149.5,142.8,138.1$, $137.2,135.1,129.9,129.5,128.1,128.1,127.0,126.9$, 126.6, 125.8, 121.5, 120.2, 56.6, 29.1, 16.8; FT-IR (film) $v$ : $3050,3023,1592,1454,1238,1027 \mathrm{~cm}^{-1}$; HRMS [APCI] calcd for $\mathrm{C}_{24} \mathrm{H}_{21}[\mathrm{M}+\mathrm{H}]^{+}$309.1638, found 309.1640.

5'-溴-2',3'-二苯基螺[环丁烷-1,1'-狮](5b): 白色固体, 产率 67\%. m.p. $140 \sim 141{ }^{\circ} \mathrm{C} ;{ }^{1} \mathrm{H}$ NMR $(400 \mathrm{MHz}$, $\left.\mathrm{CDCl}_{3}\right) \delta: 7.72 \sim 7.67(\mathrm{~m}, 1 \mathrm{H}), 7.57 \sim 7.44(\mathrm{~m}, 2 \mathrm{H})$, $7.44 \sim 7.35(\mathrm{~m}, 3 \mathrm{H}), 7.34 \sim 7.22(\mathrm{~m}, 7 \mathrm{H}), 2.73 \sim 2.61(\mathrm{~m}$, $2 \mathrm{H}), 2.61 \sim 2.46(\mathrm{~m}, 2 \mathrm{H}), 2.30 \sim 2.16(\mathrm{~m}, 1 \mathrm{H}), 1.91 \sim 1.77$ $(\mathrm{m}, 1 \mathrm{H}) ;{ }^{13} \mathrm{C}$ NMR $\left(100 \mathrm{MHz}, \mathrm{CDCl}_{3}\right) \delta: 150.5,150.4$, $144.5,136.9,136.1,133.9,129.2,128.8,127.8,127.7$, 127.7, 126.8, 126.7, 122.8, 122.4, 120.0, 55.9, 28.3, 16.2; FT-IR (film) $v: 3054,2979,1591,1454,1239,1072 \mathrm{~cm}^{-1}$; HRMS [APCI] calcd for $\mathrm{C}_{24} \mathrm{H}_{20} \mathrm{Br}[\mathrm{M}+\mathrm{H}]^{+}$387.0748, found 387.0752 .

2',3'-二(4-甲氧基苯基)螺 [环丁烷-1,1'-狮](5c): 白色 固体, 产率 43\%. m.p. $137 \sim 139{ }^{\circ} \mathrm{C} ;{ }^{1} \mathrm{H}$ NMR $(400 \mathrm{MHz}$, 
$\left.\mathrm{CDCl}_{3}\right) \delta: 7.83 \sim 7.76(\mathrm{~m}, 1 \mathrm{H}), 7.35 \sim 7.31(\mathrm{~m}, 2 \mathrm{H})$, $7.30 \sim 7.27(\mathrm{~m}, 1 \mathrm{H}), 7.24 \sim 7.18(\mathrm{~m}, 4 \mathrm{H}), 6.92 \sim 6.86(\mathrm{~m}$, $2 \mathrm{H}), 6.84 \sim 6.80(\mathrm{~m}, 2 \mathrm{H}), 3.83(\mathrm{~s}, 3 \mathrm{H}), 3.79(\mathrm{~s}, 3 \mathrm{H}), 2.67 \sim$ $2.58(\mathrm{~m}, 2 \mathrm{H}), 2.57 \sim 2.48(\mathrm{~m}, 2 \mathrm{H}), 2.26 \sim 2.15(\mathrm{~m}, 1 \mathrm{H})$, $1.91 \sim 1.79(\mathrm{~m}, 1 \mathrm{H}) ;{ }^{13} \mathrm{C}$ NMR $\left(100 \mathrm{MHz}, \mathrm{CDCl}_{3}\right) \delta$ : $158.5,158.3,152.4,148.5,143.0,137.4,131.0,130.6$, $129.5,127.6,126.4,125.5,121.3,120.1,113.6,113.5$, 56.4, 55.2, 55.1, 29.1, 16.7; FT-IR (film) v: 3002, 2935, 1574, 1503, 1458, $1239 \mathrm{~cm}^{-1}$; HRMS [APCI] calcd for $\mathrm{C}_{26} \mathrm{H}_{25} \mathrm{O}_{2}[\mathrm{M}+\mathrm{H}]^{+}$369.1855, found 369.1861.

2',3'-二(4-氟苯基)螺 [环丁烷-1,1'-狮](5d): 白色固 体, 产率 52\%. m.p. 164 165 ${ }^{\circ} \mathrm{C} ;{ }^{1} \mathrm{H}$ NMR (400 MHz, $\left.\mathrm{CDCl}_{3}\right) \delta: 7.82 \sim 7.77(\mathrm{~m}, 1 \mathrm{H}), 7.38 \sim 7.33(\mathrm{~m}, 1 \mathrm{H})$, $7.31 \sim 7.27(\mathrm{~m}, 2 \mathrm{H}), 7.25 \sim 7.19(\mathrm{~m}, 4 \mathrm{H}), 7.08 \sim 7.02(\mathrm{~m}$, $2 \mathrm{H}), 7.00 \sim 6.93(\mathrm{~m}, 2 \mathrm{H}), 2.62 \sim 2.50(\mathrm{~m}, 4 \mathrm{H}), 2.26 \sim 2.14$ $(\mathrm{m}, 1 \mathrm{H}), 1.86 \sim 1.74(\mathrm{~m}, 1 \mathrm{H}) ;{ }^{13} \mathrm{C}$ NMR $(100 \mathrm{MHz}$, $\left.\mathrm{CDCl}_{3}\right) \delta: 162.7(\mathrm{~d}, J=30.7 \mathrm{~Hz}), 160.2(\mathrm{~d}, J=30.8 \mathrm{~Hz})$, 151.5, 148.1, 141.9, 137.1, $132.3(\mathrm{~d}, J=3.5 \mathrm{~Hz}), 130.9$ (d, $J=7.9 \mathrm{~Hz}), 130.5(\mathrm{~d}, J=7.8 \mathrm{~Hz}), 130.3(\mathrm{~d}, J=3.3 \mathrm{~Hz})$, 126.2, 125.5, 121.0, 119.6, 114.9 (d, $J=15.6 \mathrm{~Hz}), 114.7$ (d, $J=15.7 \mathrm{~Hz}), 56.0,28.5,16.2 ;{ }^{19} \mathrm{~F}$ NMR $(376 \mathrm{MHz}$, $\left.\mathrm{CDCl}_{3}\right) \delta:-114.8(\mathrm{~s}),-115.0$ (s); FT-IR (film) $v: 3066$, 2991, 1596, 1508, 1353, $1273 \mathrm{~cm}^{-1}$; HRMS [APCI] calcd for $\mathrm{C}_{24} \mathrm{H}_{19} \mathrm{~F}_{2}[\mathrm{M}+\mathrm{H}]^{+}$345.1455, found 345.1461.

$2^{\prime}, 3^{\prime}$-二(4-氯苯基)螺 [环丁烷-1,1'-狮](5e): 白色固 体, 产率 51\%. m.p. 169 170 ${ }^{\circ} \mathrm{C} ;{ }^{1} \mathrm{H}$ NMR $(400 \mathrm{MHz}$, $\left.\mathrm{CDCl}_{3}\right) \delta: 7.85 \sim 7.79(\mathrm{~m}, 1 \mathrm{H}), 7.41 \sim 7.32(\mathrm{~m}, 3 \mathrm{H})$, $7.31 \sim 7.26(\mathrm{~m}, 4 \mathrm{H}), 7.25 \sim 7.14(\mathrm{~m}, 4 \mathrm{H}), 2.72 \sim 2.45(\mathrm{~m}$, $4 \mathrm{H}), 2.29 \sim 2.16(\mathrm{~m}, 1 \mathrm{H}), 1.90 \sim 1.77(\mathrm{~m}, 1 \mathrm{H}) ;{ }^{13} \mathrm{C} \mathrm{NMR}$ $\left(100 \mathrm{MHz}, \mathrm{CDCl}_{3}\right) \delta: 151.6,148.1,141.6,137.2,134.8$, $132.8,132.7,132.4,130.6,130.2,128.1,128.0,126.2$, 125.7, 121.1, 119.6, 56.1, 28.5, 16.2; FT-IR (film) v: 3019, 2935, 1491, 1239, 1089, $1014 \mathrm{~cm}^{-1}$; HRMS [APCI] calcd for $\mathrm{C}_{24} \mathrm{H}_{19} \mathrm{Cl}_{2}[\mathrm{M}+\mathrm{H}]^{+}$377.0864, found 377.0865.

2',3'-二(4-溴苯基)螺[环丁烷-1,1'-茚](5f): 白色固体, 产率 73\%. m.p. $146 \sim 147{ }^{\circ} \mathrm{C} ;{ }^{1} \mathrm{H}$ NMR $(400 \mathrm{MHz}$, $\left.\mathrm{CDCl}_{3}\right) \delta: 7.80(\mathrm{~d}, J=7.6 \mathrm{~Hz}, 1 \mathrm{H}), 7.51 \sim 7.46(\mathrm{~m}, 2 \mathrm{H})$, $7.44 \sim 7.39(\mathrm{~m}, 2 \mathrm{H}), 7.38 \sim 7.33(\mathrm{~m}, 1 \mathrm{H}), 7.30 \sim 7.27(\mathrm{~m}$, 2H), $7.16 \sim 7.10(\mathrm{~m}, 4 \mathrm{H}), 2.61 \sim 2.50(\mathrm{~m}, 4 \mathrm{H}), 2.25 \sim 2.16$ $(\mathrm{m}, 1 \mathrm{H}), 1.88 \sim 1.76(\mathrm{~m}, 1 \mathrm{H}) ;{ }^{13} \mathrm{C}$ NMR $(100 \mathrm{MHz}$, $\left.\mathrm{CDCl}_{3}\right) \delta: 152.1,148.5,142.0,137.6,135.7,133.7,131.6$, $131.5,131.4,131.0,126.7,126.2,121.6,121.5,121.2$, 120.1, 56.6, 29.0, 16.7; FT-IR (film) v: 3061, 2929, 1480, 1463, 1069, $1009 \mathrm{~cm}^{-1}$; HRMS [APCI] calcd for $\mathrm{C}_{24} \mathrm{H}_{19} \mathrm{Br}_{2} \quad[\mathrm{M}+\mathrm{H}]^{+}$464.9853, found 464.9868 .
2',3'-二(3-氟苯基)螺 [环丁烷-1,1'-狮](5g)：白色固 体, 产率 $42 \%$. m.p. $151 \sim 152{ }^{\circ} \mathrm{C} ;{ }^{1} \mathrm{H}$ NMR $(400 \mathrm{MHz}$, $\left.\mathrm{CDCl}_{3}\right) \delta: 7.82 \sim 7.77(\mathrm{~m}, 1 \mathrm{H}), 7.39 \sim 7.27(\mathrm{~m}, 4 \mathrm{H})$, $7.24 \sim 7.20(\mathrm{~m}, 1 \mathrm{H}), 7.05 \sim 6.88(\mathrm{~m}, 6 \mathrm{H}), 2.63 \sim 2.49(\mathrm{~m}$, $4 \mathrm{H}), 2.25 \sim 2.14(\mathrm{~m}, 1 \mathrm{H}), 1.87 \sim 1.75(\mathrm{~m}, 1 \mathrm{H}) ;{ }^{13} \mathrm{C} \mathrm{NMR}$ $\left(150 \mathrm{MHz}, \mathrm{CDCl}_{3}\right) \delta: 163.4(\mathrm{~d}, J=4.2 \mathrm{~Hz}), 161.8(\mathrm{~d}, J=$ $3.6 \mathrm{~Hz}), 151.9,148.6,141.9,138.9$ (d, $J=7.8 \mathrm{~Hz}), 137.6$ (d, $J=1.2 \mathrm{~Hz}), 136.9$ (d, $J=8.0 \mathrm{~Hz}), 129.7$ (d, $J=8.6 \mathrm{~Hz})$, $129.6(\mathrm{~d}, J=8.4 \mathrm{~Hz}), 126.7,126.1,125.6(\mathrm{~d}, J=2.6 \mathrm{~Hz})$, $125.1(\mathrm{~d}, J=2.6 \mathrm{~Hz}), 121.5,120.2,116.6(\mathrm{~d}, J=21.2 \mathrm{~Hz})$, 116.1 (d, $J=21.6 \mathrm{~Hz}), 114.2(\mathrm{~d}, J=20.9 \mathrm{~Hz}), 114.1$ (d, $J=$ $20.9 \mathrm{~Hz}), 56.6,29.0,16.7 ;{ }^{19} \mathrm{~F} \mathrm{NMR}\left(376 \mathrm{MHz}, \mathrm{CDCl}_{3}\right) \delta$ : -113.1 (s), -113.3 (s); FT-IR (film) v: 3063, 2952, 1579, $1428,1263,1181 \mathrm{~cm}^{-1}$; HRMS [APCI] calcd for $\mathrm{C}_{24} \mathrm{H}_{19} \mathrm{~F}_{2}$ $[\mathrm{M}+\mathrm{H}]^{+} 345.1455$, found 345.1462 .

2',3'-二乙基螺 [环丁烷-1,1'-狮](5h): 无色液体, 产 率 64\%. ${ }^{1} \mathrm{H}$ NMR $\left(400 \mathrm{MHz}, \mathrm{CDCl}_{3}\right) \delta: 7.43 \sim 7.39(\mathrm{~m}$, $1 \mathrm{H}), 7.37 \sim 7.29(\mathrm{~m}, 2 \mathrm{H}), 7.23 \sim 7.18(\mathrm{~m}, 1 \mathrm{H}), 2.65 \sim 2.53$ $(\mathrm{m}, 2 \mathrm{H}), 2.51 \sim 2.42(\mathrm{~m}, 2 \mathrm{H}), 2.28(\mathrm{q}, J=7.2 \mathrm{~Hz}, 2 \mathrm{H})$, 2.23 (q, $J=7.2 \mathrm{~Hz}, 2 \mathrm{H}), 1.98 \sim 1.87(\mathrm{~m}, 1 \mathrm{H}), 1.85 \sim 1.78$ (m, 1H), 1.02 (t, $J=7.2 \mathrm{~Hz}, 3 \mathrm{H}), 1.00$ (t, $J=7.2 \mathrm{~Hz}, 3 \mathrm{H}$ ); ${ }^{13} \mathrm{C} \mathrm{NMR}\left(100 \mathrm{MHz}, \mathrm{CDCl}_{3}\right) \delta: 147.2,143.6,133.5,128.3$, 126.0, 125.9, 52.0, 35.5, 29.8, 26.1, 16.2, 13.4, 12.3; FT-IR (film) $v: 2965,2936,1708,1458,1375,1157 \mathrm{~cm}^{-1}$; HRMS [APCI] calcd for $\mathrm{C}_{16} \mathrm{H}_{21}[\mathrm{M}+\mathrm{H}]^{+}$213.1643, found 213.1649.

4',5'-二苯基螺 [环丁烷-1,6'-环戊烯并 $[b]$ 噻吩](5i): 白色固体, 产率 $35 \%$. m.p. $134 \sim 135{ }^{\circ} \mathrm{C} ;{ }^{1} \mathrm{H}$ NMR (400 $\left.\mathrm{MHz}, \mathrm{CDCl}_{3}\right) \delta: 7.43 \sim 7.32(\mathrm{~m}, 6 \mathrm{H}), 7.32 \sim 7.26(\mathrm{~m}, 3 \mathrm{H})$, $7.25 \sim 7.16(\mathrm{~m}, 3 \mathrm{H}), 2.68 \sim 2.57(\mathrm{~m}, 2 \mathrm{H}), 2.40 \sim 2.25(\mathrm{~m}$, $3 \mathrm{H}), 1.84 \sim 1.74(\mathrm{~m}, 1 \mathrm{H}) ;{ }^{13} \mathrm{C} \mathrm{NMR}\left(100 \mathrm{MHz}, \mathrm{CDCl}_{3}\right) \delta$ : $155.3,148.2,142.6,137.4,134.7,134.0,130.0,128.5$, 128.3, 128.2, 127.2, 127.2, 125.3, 120.6, 55.4, 28.4, 17.4; FT-IR (film) v: 2992, 2923, 1497, 1338, 1129, $1184 \mathrm{~cm}^{-1}$; HRMS [APCI] calcd for $\mathrm{C}_{22} \mathrm{H}_{19} \mathrm{~S}[\mathrm{M}+\mathrm{H}]^{+} 315.1207$, found 315.1208 .

2',3'-二苯基螺环己烷-1,1'-苑](5j): 白色固体, 产率 $80 \%$. m.p. $172 \sim 173{ }^{\circ} \mathrm{C} ;{ }^{1} \mathrm{H}$ NMR (400 MHz, $\left.\mathrm{CDCl}_{3}\right) \delta$ : $7.95(\mathrm{~d}, J=7.2 \mathrm{~Hz}, 1 \mathrm{H}), 7.44 \sim 7.38(\mathrm{~m}, 1 \mathrm{H}), 7.36 \sim 7.19$ (m, $10 \mathrm{H}), 7.16 \sim 7.12(\mathrm{~m}, 2 \mathrm{H}), 2.15 \sim 2.00(\mathrm{~m}, 2 \mathrm{H}), 1.95 \sim$ $1.87(\mathrm{~m}, 3 \mathrm{H}), 1.80 \sim 1.70(\mathrm{~m}, 2 \mathrm{H}), 1.60 \sim 1.50(\mathrm{~m}, 2 \mathrm{H})$, $1.33 \sim 1.22(\mathrm{~m}, 1 \mathrm{H}) ;{ }^{13} \mathrm{C}$ NMR $\left(100 \mathrm{MHz}, \mathrm{CDCl}_{3}\right) \delta$ : $153.9,151.3,143.3,138.0,136.4,134.7,130.2,129.0$, $127.4,127.3,126.4,126.3,126.0,124.4,124.0,120.2$, 54.2, 31.1, 24.7, 21.9; FT-IR (film) v: 3053, 3029, 1600, 
1461, 1075, $1029 \mathrm{~cm}^{-1}$; HRMS [APCI] calcd for $\mathrm{C}_{26} \mathrm{H}_{25}$ $[\mathrm{M}+\mathrm{H}]^{+}$337.1956, found 337.1957.

2',3',4-三苯基螺 [环己烷-1,1'-狮](5k): 白色固体, 产 率 82\%. m.p. $181 \sim 182{ }^{\circ} \mathrm{C} ;{ }^{1} \mathrm{H}$ NMR $\left(400 \mathrm{MHz}, \mathrm{CDCl}_{3}\right)$ $\delta: 8.12 \sim 8.05(\mathrm{~m}, 1 \mathrm{H}), 7.50 \sim 7.45(\mathrm{~m}, 1 \mathrm{H}), 7.44 \sim 7.28$ (m, $15 \mathrm{H}), 7.24 \sim 7.21(\mathrm{~m}, 2 \mathrm{H}), 2.67 \sim 2.58(\mathrm{~m}, 1 \mathrm{H}), 2.43 \sim$ $2.32(\mathrm{~m}, 2 \mathrm{H}), 2.20 \sim 2.10(\mathrm{~m}, 2 \mathrm{H}), 2.08 \sim 2.00(\mathrm{~m}, 2 \mathrm{H})$, $1.76 \sim 1.68(\mathrm{~m}, 2 \mathrm{H}) ;{ }^{13} \mathrm{C}$ NMR $\left(100 \mathrm{MHz}, \mathrm{CDCl}_{3}\right) \delta$ : $153.9,151.4,147.4,144.0,138.9,136.6,135.1,130.8$, $129.5,128.6,128.0,128.0,127.0,126.9,126.9,126.7$, 126.2, 124.9, 124.7, 120.9, 54.1, 43.2, 31.9, 30.1; FT-IR (film) $v$ : 3054, 2930, 1598, 1440, 1358, $1068 \mathrm{~cm}^{-1}$; HRMS [APCI] calcd for $\mathrm{C}_{32} \mathrm{H}_{29}[\mathrm{M}+\mathrm{H}]^{+}$413.2269, found 413.2276.

4,4-二甲基-2',3'-二苯基螺 [环己烷-1,1'-茆](51)：白 色固体, 产率 87\%. m.p. $169 \sim 170{ }^{\circ} \mathrm{C} ;{ }^{1} \mathrm{H}$ NMR (400 $\left.\mathrm{MHz}, \mathrm{CDCl}_{3}\right) \delta: 7.90(\mathrm{~d}, J=7.6 \mathrm{~Hz}, 1 \mathrm{H}), 7.46 \sim 7.41(\mathrm{~m}$, $1 \mathrm{H}), 7.39 \sim 7.27(\mathrm{~m}, 9 \mathrm{H}), 7.26 \sim 7.23(\mathrm{~m}, 1 \mathrm{H}), 7.22 \sim 7.19$ (m, 2H), 2.17 (ddd, $J=13.6,13.6,4.0 \mathrm{~Hz}, 2 \mathrm{H}$ ), 2.04 (ddd, $J=13.6,13.6,3.6 \mathrm{~Hz}, 2 \mathrm{H}), 1.52 \sim 1.40(\mathrm{~m}, 4 \mathrm{H}), 1.16(\mathrm{~s}$, $3 \mathrm{H}), 0.85(\mathrm{~s}, 3 \mathrm{H}) ;{ }^{13} \mathrm{C} \mathrm{NMR}\left(100 \mathrm{MHz}, \mathrm{CDCl}_{3}\right) \delta: 153.7$, $151.4,143.2,138.1,136.4,134.7,130.1,129.0,127.5$, 127.3, 126.4, 126.3, 126.0, 124.0, 124.0, 120.2, 53.9, 34.9, 32.2, 28.8, 27.0, 24.3; FT-IR (film) $v: 3081,2942,1494$, $1440,1344,1200 \mathrm{~cm}^{-1}$; HRMS [APCI] calcd for $\mathrm{C}_{28} \mathrm{H}_{29}$ $[\mathrm{M}+\mathrm{H}]^{+}$365.2269, found 365.2273.

3,3,5,5-四甲基-2',3'-二苯基螺 [环已烷-1,1'-狮](5m): 白色固体, 产率 $46 \%$. m.p. $122 \sim 123{ }^{\circ} \mathrm{C} ;{ }^{1} \mathrm{H}$ NMR $(400$ $\left.\mathrm{MHz}, \mathrm{CDCl}_{3}\right) \delta: 7.69 \sim 7.62(\mathrm{~m}, 1 \mathrm{H}), 7.32 \sim 7.21(\mathrm{~m}$, $10 \mathrm{H}), 7.20 \sim 7.16(\mathrm{~m}, 3 \mathrm{H}), 2.20(\mathrm{~d}, J=14.4 \mathrm{~Hz}, 2 \mathrm{H}), 1.67$ (d, $J=14.0 \mathrm{~Hz}, 1 \mathrm{H}), 1.48(\mathrm{~d}, J=15.2 \mathrm{~Hz}, 2 \mathrm{H}), 1.40$ (d, $J=$ $14.0 \mathrm{~Hz}, 1 \mathrm{H}), 1.07(\mathrm{~s}, 6 \mathrm{H}), 0.77(\mathrm{~s}, 6 \mathrm{H}) ;{ }^{13} \mathrm{C}$ NMR $(100$ $\left.\mathrm{MHz}, \mathrm{CDCl}_{3}\right) \delta: 156.0,154.6,143.3,138.1,136.9,135.2$, $131.1,129.4,127.9,127.7,126.9,126.7,125.9,124.9$, $124.7,120.5,56.3,50.1,39.9,34.1,32.5,30.5$; FT-IR (film) $v$ : 2989, 2897, 1470, 1438, 1364, $1028 \mathrm{~cm}^{-1}$; HRMS [APCI] calcd for $\mathrm{C}_{30} \mathrm{H}_{33}[\mathrm{M}+\mathrm{H}]^{+}$393.2582, found 393.2582 .

$(1 R, 3 S, 5 r, 7 r)-2^{\prime}, 3^{\prime}$ - 二苯基螺 [ 金刚烷-2,1'- 狮 ] (5n): 白色固体, 产率 93\%. m.p. $135 \sim 136{ }^{\circ} \mathrm{C} ;{ }^{1} \mathrm{H}$ NMR (400 $\left.\mathrm{MHz}, \mathrm{CDCl}_{3}\right) \delta: 7.29 \sim 7.27(\mathrm{~m}, 1 \mathrm{H}), 7.23 \sim 7.15(\mathrm{~m}, 6 \mathrm{H})$, $7.15 \sim 7.12(\mathrm{~m}, 1 \mathrm{H}), 7.11 \sim 7.05(\mathrm{~m}, 1 \mathrm{H}), 7.03 \sim 6.96(\mathrm{~m}$, $3 \mathrm{H}), 6.80 \sim 6.73(\mathrm{~m}, 2 \mathrm{H}), 3.20(\mathrm{t}, J=4.4 \mathrm{~Hz}, 1 \mathrm{H}), 2.92(\mathrm{~d}$, $J=2.4 \mathrm{~Hz}, 1 \mathrm{H}), 2.68 \sim 2.62(\mathrm{~m}, 1 \mathrm{H}), 2.40 \sim 2.31(\mathrm{~m}, 1 \mathrm{H})$, $2.08 \sim 1.96(\mathrm{~m}, 3 \mathrm{H}), 1.96 \sim 1.86(\mathrm{~m}, 3 \mathrm{H}), 1.86 \sim 1.80(\mathrm{~m}$,
1H), $1.79 \sim 1.69(\mathrm{~m}, 3 \mathrm{H}) ;{ }^{13} \mathrm{C}$ NMR $\left(100 \mathrm{MHz}, \mathrm{CDCl}_{3}\right) \delta$ : $146.0,142.4,141.8,137.5,136.4,128.1,127.8,127.5$, $127.3,127.2,127.2,126.0,125.5,125.0,59.5,50.5,48.2$, 35.4, 34.8, 32.1, 31.3, 29.2, 25.9, 25.9; FT-IR (film) $v$ : $3075,2911,1573,1442,1185,1078 \mathrm{~cm}^{-1}$; HRMS [APCI] calcd for $\mathrm{C}_{30} \mathrm{H}_{29}[\mathrm{M}+\mathrm{H}]^{+}$389.2269, found 389.2270.

1',2'-二苯基螺 [环已烷-1,3'-环戊烯并 $[a$ ]萗](5o): 白 色固体, 产率 60\%. m.p. 201 202 ${ }^{\circ} \mathrm{C} ;{ }^{1} \mathrm{H}$ NMR (400 $\left.\mathrm{MHz}, \mathrm{CDCl}_{3}\right) \delta: 8.11(\mathrm{~d}, J=8.4 \mathrm{~Hz}, 1 \mathrm{H}), 7.88(\mathrm{~d}, J=8.4$ $\mathrm{Hz}, 1 \mathrm{H}), 7.77(\mathrm{~d}, J=8.4 \mathrm{~Hz}, 1 \mathrm{H}), 7.41(\mathrm{~d}, J=8.8 \mathrm{~Hz}, 1 \mathrm{H})$, 7.34 (ddd, $J=8.0 .6 .8,0.8 \mathrm{~Hz}, 1 \mathrm{H}), 7.28 \sim 7.22(\mathrm{~m}, 7 \mathrm{H})$, $7.21 \sim 7.16(\mathrm{~m}, 1 \mathrm{H}), 7.15 \sim 7.10(\mathrm{~m}, 1 \mathrm{H}), 7.10 \sim 7.06(\mathrm{~m}$, $2 \mathrm{H}), 2.18 \sim 2.03(\mathrm{~m}, 2 \mathrm{H}), 2.00 \sim 1.90(\mathrm{~m}, 2 \mathrm{H}), 1.91 \sim 1.84$ $(\mathrm{m}, 1 \mathrm{H}), 1.82 \sim 1.70(\mathrm{~m}, 2 \mathrm{H}), 1.66 \sim 1.60(\mathrm{~m}, 2 \mathrm{H}) 1.40 \sim$ $1.32(\mathrm{~m}, 1 \mathrm{H}) ;{ }^{13} \mathrm{C}$ NMR $\left(100 \mathrm{MHz}, \mathrm{CDCl}_{3}\right) \delta: 156.6$, $150.3,140.1,138.2,138.1,136.9,133.3,130.7,129.9$, $128.5,128.4,127.9,127.5,126.7,126.6,125.0,125.0$, 124.6, 123.1, 54.3, 31.6, 25.2, 22.5; FT-IR (film) $v: 3052$, 2916, 1514, 1460, 1234, $1071 \mathrm{~cm}^{-1}$; HRMS [APCI] calcd for $\mathrm{C}_{30} \mathrm{H}_{27}[\mathrm{M}+\mathrm{H}]^{+}$387.2113, found 387.2122.

2',3'-二苯基螺 [环庚烷-1,1'-狮](5p): 白色固体，产 率 77\%. m.p. $161 \sim 162{ }^{\circ} \mathrm{C} ;{ }^{1} \mathrm{H}$ NMR $\left(400 \mathrm{MHz}, \mathrm{CDCl}_{3}\right)$ $\delta: 7.69 \sim 7.62(\mathrm{~m}, 1 \mathrm{H}), 7.32 \sim 7.24(\mathrm{~m}, 6 \mathrm{H}), 7.24 \sim 7.20$ (m, 4H), $7.20 \sim 7.13(\mathrm{~m}, 3 \mathrm{H}), 2.14$ (dd, $J=14.8,9.6 \mathrm{~Hz}$, $2 \mathrm{H}), 1.89 \sim 1.70(\mathrm{~m}, 4 \mathrm{H}), 1.68 \sim 1.57(\mathrm{~m}, 2 \mathrm{H}), 1.53 \sim 1.36$ $(\mathrm{m}, 4 \mathrm{H}) ;{ }^{13} \mathrm{C} \mathrm{NMR}\left(150 \mathrm{MHz}, \mathrm{CDCl}_{3}\right) \delta: 154.7,154.3$, $142.9,137.4,137.4,135.0,130.3,129.4,127.8,127.7$, 126.7, 126.6, 126.4, 125.1, 122.5, 120.5, 57.8, 35.8, 31.9, 25.2; FT-IR (film) $v$ : 2925, 2851, 1466, 1439, 1075, 1030 $\mathrm{cm}^{-1}$; HRMS [APCI] calcd forC $\mathrm{C}_{27} \mathrm{H}_{27}[\mathrm{M}+\mathrm{H}]^{+} 351.2107$, found 351.2113 .

2',3'-二苯基螺 [环辛烷-1,1'-狮](5q)：白色固体, 产 率 62\%. m.p. $123 \sim 124{ }^{\circ} \mathrm{C} ;{ }^{1} \mathrm{H}$ NMR $\left(400 \mathrm{MHz}, \mathrm{CDCl}_{3}\right)$ $\delta: 7.58 \sim 7.53(\mathrm{~m}, 1 \mathrm{H}), 7.38 \sim 7.34(\mathrm{~m}, 1 \mathrm{H}), 7.33 \sim 7.31$ (m, $3 \mathrm{H}), 7.30 \sim 7.23(\mathrm{~m}, 6 \mathrm{H}), 7.23 \sim 7.18(\mathrm{~m}, 3 \mathrm{H}), 2.32 \sim$ $2.14(\mathrm{~m}, 2 \mathrm{H}), 1.89 \sim 1.71(\mathrm{~m}, 6 \mathrm{H}), 1.57 \sim 1.50(\mathrm{~m}, 2 \mathrm{H})$, $1.43 \sim 1.31(\mathrm{~m}, 4 \mathrm{H}) ;{ }^{13} \mathrm{C}$ NMR $\left(150 \mathrm{MHz}, \mathrm{CDCl}_{3}\right) \delta$ : $155.9,151.6,143.4,137.8,137.0,135.0,130.1,129.5$, $127.8,127.6,126.7,126.6,126.4,124.8,123.6,120.6$, 58.2, 31.3, 29.2, 26.1, 25.2; FT-IR (film) v: 3023, 2924, 1572, 1441, 1239, $1028 \mathrm{~cm}^{-1}$; HRMS [APCI] calcd for $\mathrm{C}_{27} \mathrm{H}_{27}[\mathrm{M}+\mathrm{H}]^{+}$365.2269, found 365.2277.

2',3'-二苯基螺 [环十二烷-1,1'-狮](5r): 白色固体, 产率 91\%. m.p. $135 \sim 136{ }^{\circ} \mathrm{C} ;{ }^{1} \mathrm{H}$ NMR $(400 \mathrm{MHz}$, $\left.\mathrm{CDCl}_{3}\right) \delta: 7.56 \sim 7.50(\mathrm{~m}, 1 \mathrm{H}), 7.34 \sim 7.30(\mathrm{~m}, 1 \mathrm{H})$, 
$7.30 \sim 7.27(\mathrm{~m}, 2 \mathrm{H}), 7.26 \sim 7.24(\mathrm{~m}, 2 \mathrm{H}), 7.24 \sim 7.21(\mathrm{~m}$, $4 \mathrm{H}), 7.21 \sim 7.19(\mathrm{~m}, 3 \mathrm{H}), 7.18 \sim 7.16(\mathrm{~m}, 1 \mathrm{H}), 2.02 \sim 1.94$ $(\mathrm{m}, 2 \mathrm{H}), 1.86 \sim 1.77(\mathrm{~m}, 2 \mathrm{H}), 1.50 \sim 1.31(\mathrm{~m}, 18 \mathrm{H}) ;{ }^{13} \mathrm{C}$ NMR (150 MHz, $\left.\mathrm{CDCl}_{3}\right) \delta: 154.0,151.9,143.1,138.7$, $138.7,135.2$, 130.0, 129.6, 127.8, 127.7, 126.6, 126.5, $126.3,124.5,123.5,120.4,58.2,31.9,27.5,26.2,23.4$, 23.2, 21.3; FT-IR (film) v: 3061, 2961, 1490, 1261, 1072, $1028 \mathrm{~cm}^{-1}$; HRMS [APCI] calcd for $\mathrm{C}_{32} \mathrm{H}_{37}[\mathrm{M}+\mathrm{H}]^{+}$ 421.2895, found 421.2906 .

辅助材料(Supporting Information) 化合物 $\mathbf{3 a} \sim \mathbf{3 h}$ 和 5a $\sim 5 r$ 的 ${ }^{1} \mathrm{H}$ NMR、 ${ }^{13} \mathrm{C}$ NMR 和 ${ }^{19} \mathrm{~F} \mathrm{NMR}$ 谱图. 这些材 料可以免费从本刊网站(http://sioc-journal.cn/)上下载.

\section{References}

[1] (a) Huffman, J. W.; Padgett, L. W. Curr. Med. Chem. 2005, 12, 1395.

(b) Shin, S.; Son, J. Y.; Choi, C.; Kim, S.; Lee, P. H. J. Org. Chem. 2016, 81, 11706.

[2] Yao, X.-S.; Wang, N.-L.; Bei, Z.-G.; Liu, D.-L. CN 1594311, 2005 [Chem. Abstr. 2006, 144, 184664].

[3] (a) Herdman, C. A.; Strecker, T. E.; Tanpure, R. P.; Chen, Z.; Winters, A.; Gerberich, J.; Liu, L.; Hamel, E.; Mason, R. P.; Chaplin, D. J.; Trawick, M. L.; Pinney. Med, K. G. Med. Chem. Commun. 2016, 2418.

(b) Clegg, N. J.; Paruthiyil, S.; Leitman, D. C.; Scanlan, T. S. J. Med. Chem. 2005, 48, 5989 .

(c) Wei, R. B.; Liu, Y.; Liang, Y. Chin. J. Org. Chem. 2009, 29, 476 (in Chinese).

(魏荣宝, 刘洋, 梁娅, 有机化学, 2009, 29, 476.)

[4] (a) Femia, A. P.; Soares, P. V.; Luceri, C.; Lodovici, M.; Giannini, A.; Caderni, G. BMC Cancer 2015, 15, 1.

(b) Prade, E.; Barucker, C.; Sarkar, R.; Althoff-Ospelt, G.; Lopez, D. A.; Juan, M.; Hossain, S.; Zhong, Y.; Multhaup, G.; Reif, B. Biochemistry 2016, 55, 1839.

(c) Raz, A. Biochem. Pharmacol. 2002, 63, 343.

(d) Alt, H. G.; Koppl, A. Chem. Rev. 2000, 100, 1205.

(e) Ren, S.; Igarashi, E.; Nakajima, K.; Kanno, K.; Takahashi, T. J. Am. Chem. Soc. 2009, 131, 7492.

(f) Zargarian, D. Coord. Chem. Rev. 2002, 233, 157.

(g) Leino, R.; Lehmus, P.; Lehtonen, A. Eur. J. Inorg. Chem. 2004, 16, 3201 .

(h) Ding, Y.; Tian, J.; Zhu, N. Chin. J. Org. Chem. 2010, 30, 1156 (in Chinese).

(丁研, 田喆, 朱能, 有机化学, 2010, 30, 1156.)

[5] (a) Phatake, R. S.; Ramana, C. V. Tetrahedron Lett. 2015, 56, 2183.

(b) Phatake, R. S.; Ramana, C. V. Tetrahedron Lett. 2015, 56, 3868.

(c) Wei, R. B.; Liang, Y. Chin. J. Org. Chem. 2008, 28, 1287 (in Chinese).

(魏荣宝, 梁娅, 有机化学, 2008, 28, 1287.)

(d) Kowada, T.; Yamaguchi, S.; Ohe, K. Org. Lett. 2010, 12, 296.

(e) Wu, C. L.; Chen, C. T.; Chen, C. T. Org. Lett. 2014, 16, 2114.

[6] (a) Wang, B. Q. Coord. Chem. Rev. 2006, 250, 242.

(b) Alt, H. G.; Kçppl, A. Chem. Rev. 2000, 100, 1205

(c) Zargarian, D. Coord. Chem. Rev. 2002, 233 234, 157.

[7] (a) Hu, A.-G.; Fu, Y.; Xie, J.-H.; Zhou, H.; Wang, L.-X.; Zhou, Q.-L. Angew. Chem., Int. Ed. 2002, 41, 2348.

(b) Cheng, X.; Zhang, Q.; Xie, J.-H.; Wang, L.-X.; Zhou, Q.-L. Angew. Chem., Int. Ed. 2005, 44, 1118

(c) Jia, Y.-X.; Zhong, J.; Zhu, S.-F.; Zhang, C.-M.; Zhou, Q.-L. Angew. Chem., Int. Ed. 2007, 46, 5565. (d) Xu, B.; Li, M.-L.; Zuo, X.-D.; Zhu, S.-F.; Zhou, Q.-L. J. Am. Chem. Soc. 2015, 137, 8700 .

(e) Yang, X.-H.; Yue, H.-T.; Yu, N.; Li, Y.-P.; Xie, J.-H.; Zhou, Q.-L. Chem. Sci. 2017, 8, 1181

(f) Yang, S.; Che, W.; Wu, H.-L.; Zhu, S.-F.; Zhou, Q.-L. Chem. Sci. 2017, 8, 1977.

(g) Bao, D.-H.; Wu, H.-L.; Liu, C.-L.; Xie, J.-H.; Zhou, Q.-L. Angew. Chem., Int. Ed. 2015, 54, 8791.

[8] (a) Patureau, F. W.; Besset, T.; Kuhl, N.; Glorius, F. J. Am. Chem. Soc. 2011, 133, 2154.

(b) Tobisu, M.; Nakai, H.; Chatani, N. J. Org. Chem. 2009, 74 5471 .

(c) Miyamoto, M.; Harada, Y.; Tobisu, M.; Chatani, N. Org. Lett. 2008, 10, 2975.

(d) Jia, X. D.; Petrone, D. A.; Lautens, M. Angew. Chem., Int. Ed. 2012, 51, 9870 .

(e) Zeng, X. M.; Ilies, L.; Nakamura, E. J. Am. Chem. Soc. 2011, 133, 17638 .

(f) Zheng, H. J.; Xie, X.; Yang, J.; Zhao, C. G.; Jing, P.; Fang, B. W.; She, X. G. Org. Biomol. Chem. 2011, 9, 7755.

(g) Ye, S.; Gao, K.; Zhou, H.; Yang, X.; Wu, J. Chem. Commun. 2009, 5406.

(h) Li, C.; Wang, J. Tetrahedron Lett. 2009, 50, 2956.

[9] (a) Kinoshita, H.; Hirai, N.; Miura, K. J. Org. Chem. 2014, 79, 8171 .

(b) Wu, L.; Shi, M.; Li, Y. X. Chem.-Eur. J. 2010, 16, 5163.

(c) Niharika, P.; Satyanarayana, G. ChemistrySelect 2018, 3, 289.

(d) Wang, J. L.; Zhang, L. X.; Jing, Y. F.; Huang, W.; Zhou, X. G. Tetrahedron Lett. 2009, 50, 4978.

(e) Zhou, X. B.; Zhang, H. M.; Xie, X.; Li, Y. Z. J. Org. Chem. 2008, 73, 3958.

(f) Wang, J. L.; Zhang, L. X.; Jing, Y. F.; Huang, W.; Zhou, X. G. Tetrahedron Lett. 2009, 50, 4978

(g) Liu, C.; Zhang, H.; Ding, L.; Liu, J. Chin. J. Chem. 2018, 36 737.

(h) Shen, G.; Sun, J.; Yan, C. Chin. J. Chem. 2016, 34, 412.

[10] (a) Liu, Z. Q.; Wang, J. G.; Han, J.; Zhao, Y. K.; Zhou, B. Tetrahedron Lett. 2009, 50, 1240.

(b) Li, H.; Jin, Y.; Wang, J.; Tian, S. K. Org. Biomol. Chem. 2009 7, 3219

(c) Biswas, S.; Maiti, S.; Jana, U. Eur. J. Org. Chem. 2009, 14 2354.

(d) Ren, K.; Wang, M.; Wang. L. Eur. J. Org. Chem. 2010, 3, 565.

[11] Liu, C. R.; Yang, F. L.; Jin, Y.; Ma, X. T.; Cheng, D. J.; Li, N.; Tian, S. K. Org. Lett. 2010, 12, 3832.

[12] Bu, X. L.; Hong, J. Q.; Zhou, X. G. Adv. Synth. Catal. 2011, 353, 2111.

[13] Huang, W.; Zheng, P. Z.; Zhang, Z. X.; Liu, R. T.; Chen, Z. X.; Zhou, X. G. J. Org. Chem. 2008, 73, 6845.

[14] For reviews: (a) Fan, X.; Zhao, H.; Zhu, C. Acta Chim. Sinica 2015, 73, 979 (in Chinese).

(范雪峰, 赵会君, 朱晨, 化学学报, 2015, 73, 979.)

(b) Yan, H.; Zhu, C. Prog. Chem. 2016, 28, 1 (in Chinese).

(晏宏, 朱晨, 化学进展, 2016, 28, 1.)

(c) Ren, R.; Zhu, C. Synlett 2016, 27, 1139.

(d) Yan, H.; Zhu, C. Sci. China: Chem. 2017, 60, 214

(e) Wu, X.; Zhu, C. Chem. Rec. 2018, 18, 587.

[15] (a) Zhao, H.; Fan, X.; Yu, J.; Zhu, C. J. Am. Chem. Soc. 2015, 137, 3490 .

(b) Ren, R.; Zhao, H.; Huan, L.; Zhu, C. Angew. Chem., Int. Ed. 2015, 54, 12692 .

(c) Ren, R.; Wu, Z.; Xu, Y.; Zhu, C. Angew. Chem., Int. Ed. 2016 55, 2866.

(d) Yu, J.; Yan, H.; Zhu, C. Angew. Chem., Int. Ed. 2016, 55, 1143.

(e) Yu, J.; Zhao, H.; Liang, S.; Bao, X.; Zhu, C. Org. Biomol. Chem. 2015, 13, 7924.

(f) Fan, X.; Zhao, H.; Yu, J.; Bao, X.; Zhu, C. Org. Chem. Front. 2016, 3, 227 
(g) Ren, R.; Wu, Z.; Zhu, C. Chem. Commun. 2016, 52, 8160.

(h) Wang, D.; Ren, R.; Zhu, C. J. Org. Chem. 2016, 81, 8043.

(i) Huan, L.; Zhu, C. Org. Chem. Front. 2016, 3, 1467.

(j) Wang, M.; Wu, Z.; Zhu, C. Org. Chem. Front. 2017, 4, 427.

(k) Mao, W.; Zhu, C. J. Org. Chem. 2017, 82, 9133.
(1) Mao, W.; Zhu, C. Synlett 2018, 29, 731.

(m) Wang, D.; Mao, J.; Zhu, C. Chem. Sci. 2018, 9, 5805.

[16] (a) Souillart, L.; Cramer, N. Chem. Rev. 2015, 115, 9410.

(b) Khoury, P. R.; Goddard, J. D. Tam, W. Tetrahedron 2004, 60, 8103 .

(Cheng, F.) 